Giovanni Battista Ratti and Jorge Luis Rodríguez

\title{
On coherence as a formal property of normative systems
}

\author{
Warning \\ The contents of this site is subject to the French law on intellectual property and is the exclusive property of the \\ publisher. \\ The works on this site can be accessed and reproduced on paper or digital media, provided that they are strictly used \\ for personal, scientific or educational purposes excluding any commercial exploitation. Reproduction must necessarily \\ mention the editor, the journal name, the author and the document reference. \\ Any other reproduction is strictly forbidden without permission of the publisher, except in cases provided by legislation \\ in force in France.
}

\section{revues.org}

Revues.org is a platform for journals in the humanities and social sciences run by the CLEO, Centre for open electronic publishing (CNRS, EHESS, UP, UAPV).

\author{
Electronic reference \\ Giovanni Battista Ratti and Jorge Luis Rodríguez, « On coherence as a formal property of normative systems », \\ Revus [Online], 27 | 2015, Online since 20 August 2015, connection on 23 January 2016. URL : http:// \\ revus.revues.org/3291; DOI : 10.4000/revus.3291 \\ Publisher: Klub Revus - Center za raziskovanje evropske ustavnosti in demokracije \\ http://revus.revues.org \\ http://www.revues.org \\ Document available online on: \\ http://revus.revues.org/3291 \\ Document automatically generated on 23 January 2016. The page numbering does not match that of the print edition. \\ All rights reserved
}




\section{Giovanni Battista Ratti and Jorge Luis Rodríguez}

\section{On coherence as a formal property of normative systems}

Pages in print edition : p. _- -

\section{Introduction}

'Consistency' and 'completeness' are age-old concepts which are traditionally related to logic and logical calculi. They have received a great elaboration and their mutual relations have been widely studied in the field of logic. They have also been extensively analyzed by legal dogmatics and analytical jurisprudence regarding their application to normative systems. By contrast, 'coherence' is a relatively new concept, which has raised some issues in the epistemological and legal philosophical debate during the last decades. ${ }^{1}$ The intensional properties and the boundaries of this concept are quite blurry, and its relations with consistency and completeness are consequently uncertain. ${ }^{2}$

The present paper, by elaborating on some notions of propositional calculus, aims at providing a basic reexamination of the notions of consistency, completeness, and coherence, when applied to the normative domain, and, on such a basis, elaborating on which mutual relations they have.

\section{Consistency and Completeness in Propositional Logic}

In propositional logic, ${ }^{3}$ consistency is usually and broadly defined as the property of a set of sentences of being free of contradictions. It is not uncommon to distinguish between consistency with respect to negation and absolute consistency. A set $\mathrm{S}$ is consistent with respect to negation if and only if for every proposition $p$, if $p$ belongs to $\mathrm{S}$, then $\sim p$ does not belong to $\mathrm{S}$. By contrast, a set $\mathrm{S}$ is absolutely consistent if and only if there is at least one proposition $p$ that is not implied by $\mathbf{S}$.

$\mathrm{S}$ is consistent with respect to negation iff

$\forall p(p \in \mathrm{S} \Rightarrow \sim p \notin \mathrm{S})$

$\mathrm{S}$ is absolutely consistent iff

$\exists p(p \notin \mathrm{Cn}(\mathrm{S}))^{4}$

Two corresponding notions of inconsistency may be defined as the negations of these properties: a set $\mathrm{S}$ may be called inconsistent with respect to negation if and only if there is at least one proposition $p$ such that both $p$ and $\sim p$ belong to $\mathrm{S}$, and absolutely inconsistent if and only if every proposition $p$ is implied by $\mathrm{S}$.

$\mathrm{S}$ is inconsistent with respect to negationiff

$\sim \forall p(p \in \mathrm{S} \Rightarrow \sim p \notin \mathrm{S})=\exists p(p \in \mathrm{S} \wedge \sim p \in \mathrm{S})$

$\mathrm{S}$ is absolutely inconsistent iff

$\sim \exists p(p \notin \mathrm{Cn}(\mathrm{S}))=\forall p(p \in \mathrm{Cn}(\mathrm{S}))$

From these characterizations it is interesting to note that the properties of consistency and inconsistency with respect to negation are defined in terms of the elements that belong to a given set, while the properties of absolute consistency and inconsistency are defined in terms of the logical consequences of a given set. 
In fact, as Žarnić and Bašić (2014) claim, the notions of logical closure and absolute consistency are interdefinable according to the following equivalence:

$$
\mathrm{S}=\mathrm{Cn}(\mathrm{S}) \text { iff } \forall p(p \notin \mathrm{S} \Rightarrow(\perp \notin \mathrm{Cn}(\mathrm{S} \cup\{\sim p\}))
$$

This means that a set $\mathrm{S}$ is closed under logical consequence if and only if the negation of any element not belonging to $\mathrm{S}$ may be added to $\mathrm{S}$ preserving its absolute consistency. Completeness, in turn, is regarded as the property of a finite set of sentences (axioms) - or sentential forms (axiom schemata)—of being exhaustive qua axiomatization of a certain field, so that every correct sentence, relevant for that field, is derivable from-i.e. is a theorem of -the axiomatic basis.

It is common to distinguish between sets which are weakly complete, for they are capable of generating the set of all its correct theorems, and sets which are strongly complete, i.e. those sets which cannot be further supplemented regarding their axiomatic basis without being rendered inconsistent. To these two notions, one can add trivial completeness, the property of a set of demonstrating any sentence whatsoever. In formal terms, this translates into:

$\mathrm{S}$ is weakly complete iff $\forall p(p \in \mathrm{C} \Rightarrow p \in \mathrm{Cn}(\mathrm{S}))$

(where $\mathrm{C}$ is the set of correct or relevant sentences)

$\mathrm{S}$ is strongly complete iff $\forall p(p \notin \mathrm{S} \Rightarrow(\perp \in \mathrm{Cn}(\mathrm{S} \cup\{p\}))$

\section{$\mathrm{S}$ is trivially complete iff $\forall p(p \in \mathrm{Cn}(\mathrm{S}))^{5}$}

From these definitions it follows that a set may be called weakly incomplete if and only if at least one correct sentence is not derivable from it; strongly incomplete if and only if it can be further supplemented regarding their axiomatic basis without being rendered inconsistent, and trivially incomplete - though the label may sound a little awkward-if there is at least one sentence that it cannot demonstrate. ${ }^{6}$ In formal terms:

$\mathrm{S}$ is weakly incomplete iff

$\sim \forall p(p \in \mathrm{C} \Rightarrow p \in \mathrm{Cn}(\mathrm{S}))=\exists p(p \in \mathrm{C} \wedge p \notin \mathrm{Cn}(\mathrm{S}))$

$\mathrm{S}$ is strongly incomplete iff

$\sim \# p(p \notin \mathrm{S} \Rightarrow(\perp \in \mathrm{Cn}(\mathrm{S} \cup\{p\})))=\exists p(p \notin \mathrm{Cn}(\mathrm{S}) \wedge(\perp \notin \mathrm{Cn}(\mathrm{S} \cup\{p\})))$

$\mathrm{S}$ is trivially incomplete iff

$\sim \# p(p \in \mathrm{Cn}(\mathrm{S}))=\exists p(p \notin \mathrm{Cn}(\mathrm{S}))$

The mutual relations between these notions of consistency and completeness are as follows. First, trivial completeness is equivalent to absolute inconsistency and, consequently, trivial incompleteness is equivalent to absolute consistency. Second, trivial completeness (as well as absolute inconsistency) implies weak completeness, because if any sentence is derivable from a certain set, then the partition of all sentences that are 'correct' theorems are derivable from it. Third, if one accepts the ex falso quodlibet principle, then inconsistency with respect to negation entails absolute inconsistency (as well as trivial and weak completeness). Finally, if 
strong completeness is defined in the way proposed supra, then an absolutely inconsistent set satisfies strong completeness vacuously.

For the sake of the argument to follow in the paper, particular stress should be put on the formalization of consistency (and completeness) regarding conditional sentences. In formal logic, states of inconsistency stemming from conditional sentences are usually represented by the following conjunction of a conditional with the corresponding denied conditional:

$$
(p \Rightarrow q) \wedge \sim(p \Rightarrow q)
$$

Which, by definition of the conditional $((p \Rightarrow q) \Leftrightarrow \sim(p \wedge \sim q))$, is equivalent to:

$$
\sim(p \wedge \sim q) \wedge(p \wedge \sim q)
$$

From [1], or [2], any sentence whatsoever follows under the assumption of the ex falso quodlibet principle. In such a case, the resulting set is weakly and trivially complete. By contrast, the following sentence:

$$
(p \Rightarrow q) \wedge(p \Rightarrow \sim q)
$$

brings about no contradiction, despite what may appear at first sight. The conjunction of a conditional with its corresponding conditional denial is materially equivalent to the negation of the common antecedent of both conditionals.

[4]

$$
(p \Rightarrow q) \wedge(p \Rightarrow \sim q) \Leftrightarrow \sim p
$$

This happens since a conditional and its conditional denial are subcontraries; they are consequently neither contraries nor contradictories. Indeed, a conditional is contradictory to its negation, namely the conjunction of its antecedent and the negation of its consequent. This in turn is contrary to the conjunction of the antecedent and the consequent of the original conditional. These relations are expressed in the following square of propositional opposition:

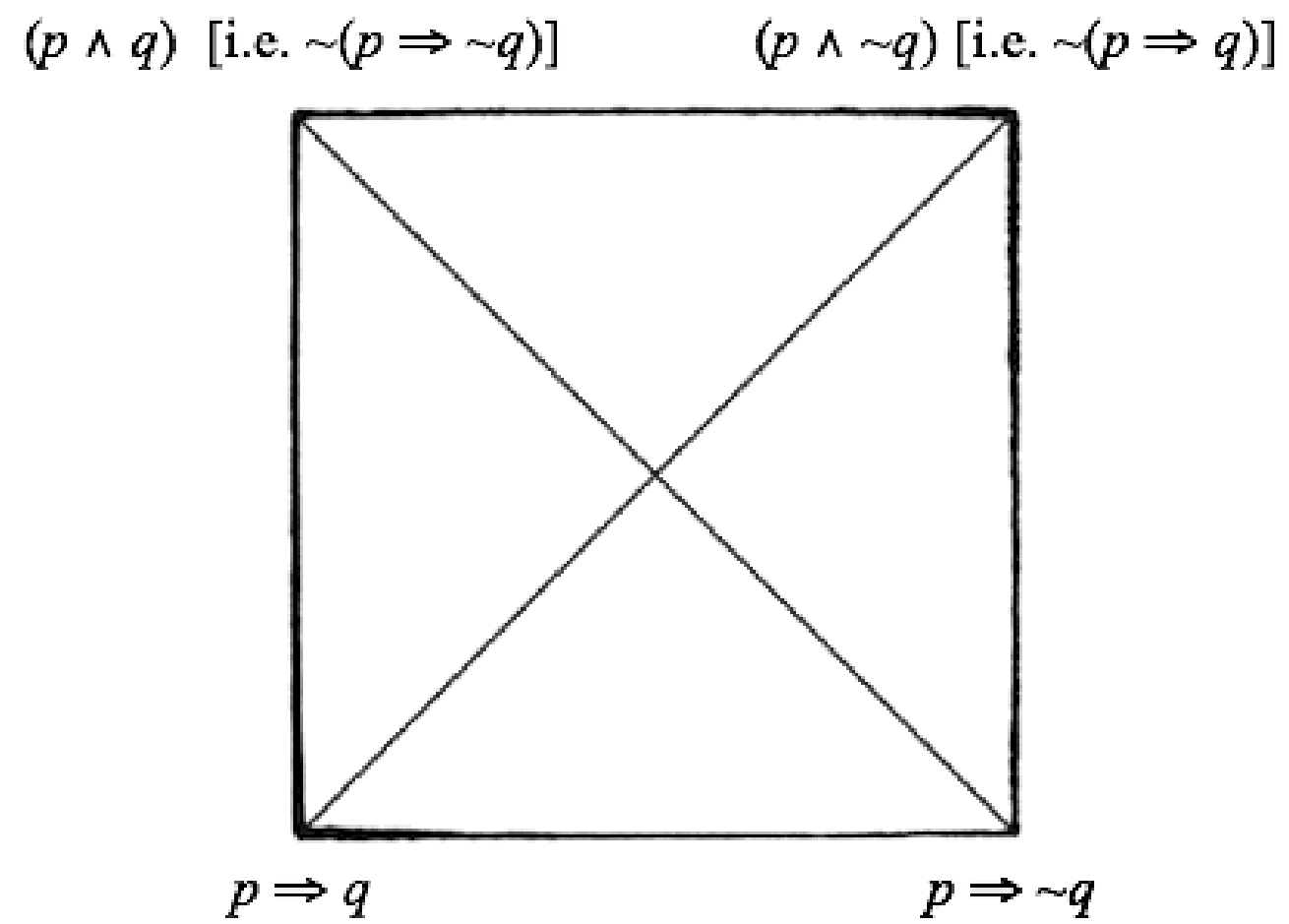

On the top side, there is contrariety: the conjunctions that appear there (which are equivalent to denied conditionals) cannot both be true, but they can both be false. On diagonals, there 
is contradiction: if one sentence is true, the other is false (and vice versa). On the verticals, there is subalternation (viz. implication): if the conjunction (i.e., the negation of the conditional denial) is true, then the corresponding conditional is also true. On the basis of the square, there is subcontrariety, which states that such conditionals can both be true, but they cannot both be false: indeed, when one of the two is false, the other is necessarily true, but when one of the two is true, it is not the case that the other is necessarily false.

When contradictions or contrarieties are contained by a certain set, this set becomes of course inconsistent, and also trivially and weakly complete. But when subcontrary sentences like ( $p$ $\Rightarrow q)$ and $(p \Rightarrow \sim q)$ are contained by a certain set, such set will not necessarily be inconsistent for that reason. It will be strongly complete regarding those worlds in which $p$ is true, since $p$ cannot be added to the set without generating an inconsistency.

Indeed, the subcontrariety of conditionals is exhaustive of a certain world, in the sense that it connects a certain atomic sentence (in our example, $p$ ) to a partition of sentences which are mutually exclusive and jointly exhaustive (in our example, $q$ and $\sim q$ ). When both conditionals are simultaneously asserted (and hence the sentence $p$ is connected, at the same time, to $q$ and $\sim q$ ), one can derive the falsity of $p$ and, as a consequence, the truth of $\sim p$.

\section{Consistency and Completeness in Normative Systems}

In the legal philosophical domain, consistency and completeness have been frequently examined. In the most relevant literature, 'normative consistency' denotes the situation where the same set of operative facts is not connected to incompatible normative consequences, whereas 'normative completeness' denotes the situation where there is a legal consequence for any set of relevant operative facts. ${ }^{7}$ Nevertheless, formal logical relations between normative consistency and completeness have seldom been taken into account. ${ }^{8}$ In order to do so, it is necessary to distinguish genuine norms from propositions about norms. ${ }^{9}$

Though the thesis that norms are proposition-like entities and, as such, are apt to truth-values, is highly controversial, there is no disputing the propositional character of the discourse about norms. Von Wright characterizes norm-propositions as propositions 'that such and such a norm exists'. ${ }^{10}$ But since norms usually exist as a part of complex systems of norms, the syntactical form of deontic statements expressing norm-propositions often hides a relevant part of their conceptual content. ${ }^{11}$ Thus, a statement like 'Parking is not allowed here', understood as a norm-proposition, though apparently referring to the deontic status of an action without qualifications, is in fact a relative statement that reports the deontic status of an action according to a particular normative system. Norm-propositions are thus true or false depending on the set of norms taken as relevant. Therefore, the structure of norm-propositions may be represented as ' $O p \in \mathrm{S}$ ', where $O p$ is the norm that is being mentioned (not used) by this norm-proposition, and $\mathrm{S}$ is the normative system to which, according to the proposition at hand, $O p$ is said to belong.

The standard system of deontic logic validates the principle of normative consistency, namely, that no action can be both prohibited and permitted, and the principle of normative completeness, namely, that every action is either prohibited or permitted. Now, as Alchourrón and Bulygin have shown, it is wrong to assume that logical relations among norm-propositions are equivalent to those that hold among norms themselves. ${ }^{12}$ A simple way to explain the differences between a genuine logic of norms and a logic of norm-proposition is to take due notice of the different ways in which negation affects the former and the latter. When applied to norms, negation seems to behave in close analogy to descriptive discourse: the negation of a norm (e.g. $O p)$ is also a norm $(P \sim p),{ }^{13}$ for each norm there is only one norm that is its negation; a norm and its negation are reciprocal (if $P \sim p$ is the negation of $O p$, then $O p$ is the negation of $P \sim p) .{ }^{14}$ Moreover, a norm and its negation are mutually exclusive and jointly exhaustive, since $\sim(O p \wedge P \sim p)$ and $O p \vee P \sim p$ are valid formulas in a logic of norms. By contrast, the negation of norm-propositions is more problematic. 'In normative system $S$ act $p$ is forbidden' is a complex metalinguistic statement that asserts that a certain norm belongs to a normative system. Therefore, its negation can be understood in two different ways: 'In system $S$ act $p$ is not forbidden' may be read as expressing that $\mathrm{S}$ does not contain a norm 
prohibiting $p$, in which case the negation operates over the membership of the norm in the system (external negation, represented as ' '); or it may be read as expressing that $S$ does contain a norm not-prohibiting $p$ (i.e. a norm permitting $p$ ), in which case the negation affects the norm referred to in the statement (internal negation, represented as ' $\neg$ '). While external negation of a norm-proposition is an operation that switches the value of membership of a given norm within a normative system, internal negation of a norm-proposition is an operation that leads to another norm-proposition asserting the membership in the system of the negation of the original norm. For example:

External negation: $\sim F_{\mathrm{S}} p=_{\text {df. }} F p \notin \mathrm{S}$

Internal negation: $\neg F_{\mathrm{S}} p=_{\text {df. }} \sim F p \in \mathrm{S}=_{\text {df. }} P p \in \mathrm{S}$

Here, the external negation of the proposition according to which $p$ is forbidden in normative system $\mathrm{S}$ is a proposition that negates the membership of a norm prohibiting $p$ in system $\mathrm{S}$, whilst the internal negation of such a proposition is a proposition asserting the membership in $\mathrm{S}$ of a norm to the effect that $p$ is not prohibited, i.e. a norm permitting $p$. Accordingly, 'permission' is an expression that may appear in a genuine norm, or in a norm-proposition. In the first case, i.e. under a prescriptive interpretation, to say that an action is permitted is unambiguously equivalent to saying that it is not prohibited. By contrast, when 'permission' appears in a norm-proposition it becomes ambiguous, since there are two alternative senses in which an action can be said to be permitted according to a set of norms: a negative sense - there is no norm in the set prohibiting it - and a positive sense - there is a norm in the set permitting it. ${ }^{15}$ We may call these two notions negative permission $\left(P^{-}{ }_{s} p\right)$ and positive permission $\left(P^{+}{ }_{\mathrm{s}} p\right)$ :

Negative permission: $P^{-}{ }_{\mathrm{s}} p={ }_{\mathrm{df} .}{ }^{F p} \notin \mathrm{S}$

Positive permission: $P^{+}{ }_{\mathrm{s}} p={ }_{\mathrm{df} .} \sim F p \in \mathrm{S}=_{\mathrm{df}} . P p \in \mathrm{S}$

It is easy to see that the negative permission of $p$ is equivalent to the external negation of the prohibition of $p$, while the positive permission of $p$ is equivalent to the internal negation of the prohibition of $p$.

The first presentation of the differences between a genuine logic of norms and a logic of normpropositions was put forward by Carlos Alchourrón, and later developed in work co-authored with Eugenio Bulygin. ${ }^{16}$ Their proposal can be set out axiomatically as follows:

\section{System of logic of norm-propositions (LNP)}

$\left(\mathrm{LNP} \mathrm{A}_{1}\right) O_{\mathrm{S}}(\alpha \wedge \beta) \#\left(O_{\mathrm{S}} \alpha \wedge O_{\mathrm{S}} \beta\right)$

(LNP A $\left.\mathrm{A}_{2}\right) O_{\mathrm{s}} \alpha \Rightarrow P_{\mathrm{s}}^{+} \alpha$

$\left(\mathrm{LNP} \mathrm{A_{3 }}\right) P^{+}{ }_{\mathrm{s}}(\alpha \wedge \beta) \Rightarrow P^{+}{ }_{\mathrm{s}} \alpha$

(LNP RI $\left.{ }_{1}\right)$ From $\vdash(\alpha \Leftrightarrow \beta)$, it follows $\vdash\left(O_{\mathrm{S}} \alpha \Leftrightarrow O_{\mathrm{S}} \beta\right)$

(LNP RI $\left.{ }_{2}\right)$ From $\vdash(\alpha \Leftrightarrow \beta)$, it follows $\vdash\left(P^{+}{ }_{\mathrm{s}} \alpha \Leftrightarrow P^{+}{ }_{\mathrm{s}} \beta\right)^{17}$

If we compare this system with the standard system of deontic logic, ${ }^{18}$ we should notice first that norm-propositions are relative to a given normative system, and that is reflected in the subscripts (s) in the formulas. By contrast, the expressions of the logic of norms do not refer to any particular normative system, since they represent absolute concepts. Second, in the system of logic for norm-propositions there is nothing like the principle of $P p \Leftrightarrow \sim O \sim p^{19}$ which is accepted as valid in deontic logic. This is so because, as we have seen, there are two different forms of negation of norm-propositions, which give rise to two descriptive concepts for each 
of the deontic operators. And although external negation satisfies all the properties that we expect from ordinary negation, that is not the case with internal negation. A norm-proposition and its internal negation can both be true-in which case the system will be inconsistent-as well as both false -in which case the system will be incomplete. ${ }^{20}$ That is why the equivalence expressed in $P p \Leftrightarrow \sim O \sim p$, though valid in the logic of norms ( $\mathrm{LN}$ ), is not valid in the logic of norm-propositions.

In spite of these differences, however, it can be proven that, under certain assumptions, LNP and $\mathrm{LN}$ are equivalent, and consequently, that positive permission $P^{+}$will be equivalent to negative permission $P^{-}$. And that is so since an analogue of the principle $P p \Leftrightarrow \sim O \sim p$ is indeed valid in LNP under the conditions of consistency and completeness of the normative system taken into account:

\begin{tabular}{|l|l|l|}
\hline$[5]$ & $\sim\left(O_{\mathrm{s}} \sim p \wedge P^{+}{ }_{\mathrm{s}} p\right)$ & $($ Cons $p)$ \\
\hline$[6]$ & $O_{\mathrm{s}} \sim p \vee P_{\mathrm{s}}^{+} p$ & $\left(\mathrm{Comp}_{\mathrm{s}} p\right)$ \\
\hline
\end{tabular}

It is important to see that the conjunction of [5] and [6], i.e.

$$
\sim\left(O_{\mathrm{s}} \sim p \wedge P^{+}{ }_{\mathrm{s}} p\right) \wedge\left(O_{\mathrm{s}} \sim p \vee P^{+}{ }_{\mathrm{s}} p\right)
$$

is equivalent to

[8]

$$
P^{+} p \Leftrightarrow \sim O_{\mathrm{s}} \sim p^{21}
$$

and since by the definitions already seen, $\sim O_{\mathrm{s}} \sim p$ is equivalent to $P^{-}{ }_{\mathrm{s}} p$, then:

[9]

$$
\left(\left(\operatorname{Cons}_{\mathrm{s}} p\right) \wedge\left(\operatorname{Comp}_{\mathrm{s}} p\right)\right) \Leftrightarrow\left(P_{\mathrm{s}}^{+} p \Leftrightarrow P_{\mathrm{s}}^{-} p\right)
$$

Moreover, if one accepts that a contradiction implies everything, normative inconsistency entails normative completeness, ${ }^{22}$ and normative incompleteness entails normative consistency, what is tantamount to saying that, at least within the normative domain, implication, consistency, and completeness are unavoidably connected concepts.

Indeed, $(i)$ if a normative system $\mathrm{S}$ is inconsistent regarding a certain action $p$, it is complete regarding that same action. This is shown in the following derivation:

\begin{tabular}{|l|l|l|}
\hline$[10]$ & $O_{\mathrm{s}} \sim p \Leftrightarrow P^{+}{ }_{\mathrm{s}}$ & \\
\hline$[11]$ & $O_{\mathrm{s}} \sim p$ & by simplification in [10] \\
\hline$[12]$ & $O_{s} \sim p \vee P^{+}{ }_{s}$ & by introduction of disjunction in [11] \\
\hline$[13]$ & $\left(O_{s} \sim p \wedge P^{+}{ }_{s} p\right) \Rightarrow\left(O_{s} \sim p \vee P^{+}{ }_{s} p\right)$ & $\begin{array}{l}\text { by introduction of conditional from } \\
{[10] \text { to [12]. }}\end{array}$ \\
\hline
\end{tabular}

And (ii) If a normative system $\mathrm{S}$ is incomplete regarding a certain action $p$, then it is consistent regarding that same action. This is demonstrated by the following inference:

\begin{tabular}{|l|l|l|}
\hline$[14]$ & $\sim\left(O_{\mathrm{s} \sim p \vee P^{+}}{ }_{\mathrm{s}} p\right)$ & \\
\hline$[15]$ & $\sim O_{\mathrm{s}} \sim p \wedge \sim P^{+}{ }_{\mathrm{s}} p$ & by De Morgan in [14] \\
\hline$[16]$ & $\sim O_{s} \sim p$ & by simplification in [15] \\
\hline
\end{tabular}




\begin{tabular}{|l|l|l|}
\hline$[17]$ & $O_{S} \sim p \wedge P^{+}{ }_{S} p$ & assumption \\
\hline$[18]$ & $O_{s} \sim p$ & by simplification in [17] \\
\hline$[19]$ & $O_{S} \sim p \wedge \sim O_{s} \sim p$ & $\begin{array}{l}\text { by introduction of conjunction in [18, } \\
16]\end{array}$ \\
\hline$[20]$ & $\sim\left(O_{S} \sim p \wedge{P^{+}}{ }_{S} p\right)$ & $\begin{array}{l}\text { by introduction of negation from [17] } \\
\text { to [19]. }{ }^{23}\end{array}$ \\
\hline
\end{tabular}

In fact, these two demonstrations can be generalized in virtue of an application of the principle ex falso sequitur quodlibet as follows:

(iii) If a normative system $\mathrm{S}$ is inconsistent regarding certain action $x$, it is complete regarding any action $\left(\exists x \sim\left(\operatorname{Cons}_{s} x\right) \Rightarrow \forall x\left(\operatorname{Comp}_{s} x\right)\right)$.

(iv) If a normative system $\mathrm{S}$ is incomplete regarding certain action $x$, it is consistent regarding any action $\left(\exists x \sim\left(\operatorname{Comp}_{\mathrm{s}} x\right) \Rightarrow \forall x\left(\right.\right.$ Cons $\left.\left._{\mathrm{s}} x\right)\right){ }^{24}$

Now, let's assume, with Alchourrón and Bulygin, that legal rules are correctly represented as conditionals of the form $p \Rightarrow O q \cdot{ }^{25}$ Denying a norm of this sort would prima facie result in the following sentence:

\begin{tabular}{|l|l|}
\hline$[21]$ & $\sim(p \Rightarrow O q)$ \\
\hline
\end{tabular}

Sentence [21] can be translated-due to the definition of conditional and the interdefinability of deontic modalities-into:

$$
p \wedge P \sim q
$$

However, deontic logicians and legal theorists do not regard [21] and [22] as the negation of the conditional sentence $p \Rightarrow O q$. They rather reserve this title for the normative sentence, which is analogous to conditional denial in propositional logic, to wit:

$$
(p \Rightarrow \sim O q)
$$

Which, for the inter-definability of deontic characters, equates to

$$
(p \Rightarrow P \sim q)
$$

Hence, regarding hypothetical normative systems ${ }^{26}$ consistency and completeness are worldsensitive, in that they only apply to the world referred to by the antecedent of conditional normative sentences, whenever they are actually instantiated. Normative inconsistency via certain facts does not trivialize the system globally. In other words, the presence in a normative system of the sentences $p \Rightarrow O q$ and $p \Rightarrow \sim O q$ renders the system inconsistent with respect to negation, but only regarding those worlds in which $p$ is the case. Here, again, if we accept the principle ex falso sequitur quodlibet, the system will also be absolutely inconsistent for those worlds in which $p$ is true, and since absolute inconsistency is equivalent to trivial completeness, this latter property will also be predicable of the system regarding $p$-worlds.

Of course, all these considerations are compatible with the claim that in hypothetical normative systems, completeness and consistency are independent properties, in that a normative system correlating normative solutions to certain cases may be complete/incomplete regarding a given case and consistent/inconsistent regarding another case, as well as consistent/inconsistent regarding a given case and complete/incomplete regarding another case. 


\section{Normative Coherence}

The concept of coherence is a highly contested one. There are many conceptions of coherence, each one predicated on a web of notions which only occasionally and partially overlap. However, if one were to search for the common kernel of such conceptions, one would probably affirm that coherence is a justificatory relation among principles and a certain set of rules to which such principles supposedly underlie. ${ }^{27}$ In many cases, coherence is left at this rather pretheoretical stage, which in turn is predicated on the fuzzy way principles are defined. However, we can make a preliminary attempt to distinguish principles from rules by pointing to (a) their fundamental character; (b) their defeasibility; (c) their need of being specified prior to their application. ${ }^{28}$

Principles are fundamental in that they justify several rules, but they do need further justification. They are regarded as defeasible for their antecedents are not taken to be sufficient conditions of their consequents (what entails that they are not liable to being formalized as material conditionals). They need to be specified in the sense that they do not allow modus ponens, and must consequently be developed into rules, although there are many alternative ways of specifying a principle into an unexpressed (viz. implicit in a non-logical sense) rule. A normative system can be said to be coherent regarding a certain set $\mathrm{P}$ of underlying principles if and only if all (or most) of its rules are instances of $\mathrm{P},{ }^{29}$ in the sense that all (or most) of its rules are justified by P. According to this idea, coherence - unlike consistency and completeness - is not an all-or-nothing property, but a gradual one.

Coherence has been said to serve three main functions: ${ }^{30}$ a simplifying one, an integrating one, and a defeating one. Simplification is given by the circumstance that a certain normative system can be traced back to a limited array of underlying principles, liable to be further developed into new rules. Integration consists in the possibility of filling up gaps in the normative system by reaching out to its underlying principles, so that the norm introduced into the system to fill the gap increases the overall coherence of the normative system. Defeasance points to the fact that rules which bring about incoherent results can be revised accordingly. Nevertheless, it is important to note that there are to different ways of understanding the relation of justification between rules and principles, and accordingly two different ways of understanding the very notion of principles, that have considerable impact over these three functions of coherence. A first interpretation of the idea that principles 'justify' rules, and of conceiving the former, is to understand 'principles' as general norms that are obtained through a process of abstraction or complete induction from the rules of a given normative system. The expression 'general principles' of law or of a concrete legal institution is usually employed to refer to this notion. On this interpretation it seems perfectly sensible to maintain that principles function as a simplification of a system of rules, and that they cannot conflict with those rules. Principles so understood are not able to display either the integration or the defeasibility function, since in this sense principles do not allow deriving solutions different from those that follow from the rules, as they would be but mere reformulations of the rules, even though some authors have correctly pointed out that so inferred principles are normally used by jurists to create "new" rules by means of strengthening the antecedent. ${ }^{31}$

45 As an alternative, it could be claimed that though rules 'exemplify' principles, the latter are not mere reformulations of the former, but express values or ends presupposed by the rules. On this reading, principles may eventually allow deriving solutions for cases not regulated by the rules, so that arguments based on principles will have certain 'ampliative' character, and they may even justify solutions in conflict with the rules of the system, and thus are capable of displaying the functions of integration and defeasibility. By contrast, it is not clear whether principles, on this understanding, can work as a simplification of a system of rules, since the idea of simplification loses its proper sense if we are not dealing with axiomatic bases with identical normative consequences where one of them is more economical than the other. But here, insofar as the considered principles allow deriving normative solutions for cases which are not regulated by the rules of the system, and even normative solutions incompatible with those that follow from the rules, it is clear that the system of rules and the system of 
principles may have different consequences. And it is only here, on this second understanding of principles, that the idea of coherence of a system of rules with a set of underlying principles is significant and not merely trivial, as in the previous understanding, where rules cannot but cohere with the principles abstracted from those same rules.

It is interesting to inquire into the relations between coherence (understood as a relation between a system of rules and a system of principles) and the previously examined properties of completeness and consistency of both systems. First, the simplifying function of coherence seems to be connected to weak completeness, in that it allows one to build an axiomatic basis, liable to be developed into all its relevant consequences. However, it should be kept in mind that this function of coherence only holds in proper sense for the first understanding of principles, and that on this reading any system of rules is trivially coherent with the principles abstracted from them.

Now, on the second understanding of principles, and when it comes to the system of rules, coherence is not necessarily connected to strong completeness or consistency (at least with respect to negation) of this latter system. In fact, coherence sometimes seems to require the introduction of a new (unexpressed) rule in the system which is in conflict with an older (expressed) one: the famous case Riggs v. Palmer ${ }^{32}$ is a clear instance of this phenomenon. As everybody knows, the legal question in Riggs v. Palmer was whether Elmer Palmer, who had poisoned his grandfather, was entitled to inherit his grandfather's assets under the Statute of Wills which was in force, at that time, in the state of New York. The Statute of Wills was completely silent about unworthy beneficiaries, and only provided that one was entitled to inherit if one was so vested by a valid will. One could maintain-as Gray J. actually did in his dissenting opinion - that Elmer was entitled to inherit under both the Statute of Wills and his grandfather's will. However, the majority opinion, drafted by Earl J., offered a different solution: the U.S. legal system could not admit, from an axiological point of view, the extremely unjust result of letting the inheritance go to someone who killed his own testator. The rejection of such a solution was founded on the presumed existence, within the U.S. legal system, of the principle according to which 'No man should profit from his own wrong'. On the basis of this principle, an unexpressed rule was introduced according to which 'The unworthy beneficiary, though granted the inheritance by a valid will, is not entitled to inherit ${ }^{33}$ Manifestly, such a rule conflicts with another rule (regarded as in force until the Riggs case) according to which a valid will is sufficient in order to inherit the testator's assets. This case exemplifies a situation where the exigencies of axiological coherence brought the legal applicators to introduce a rule into the system which made it normatively inconsistent. If this is correct, coherence sometimes runs counter to the preservation of strong completeness and consistency of the system of rules, for it can encourage legal applicators to introduce rules which bring about new inconsistencies, which were not present before. Recall that strong completeness has been defined as the situation where no sentence can be added to the axiomatic basis of a certain set without rendering it inconsistent. It is easy to see that coherence of the system of rules with a set of underlying principles is an exigency which can (and do) often conflict with this ideal, for it requires the continuous introduction of new ('apocryphal') rules on the basis of axiological judgments regarding the inadequacy of the existing rules.

This last conclusion has to be balanced with another usual role assigned to principles: sometimes we appeal to a set of underlying principles to give priority to one normative solution within a set of inconsistent solutions derivable from the system of rules. In such case, principles would be used to restore consistency in the system of rules. Moreover, though this idea deserves a thorough justification that we will not try to offer here, it may be suggested that coherence with a set of underlying principles could even stop whatever consequences to follow from a normative contradiction within the system of rules, for they would not match the requirements set by those underlying principles. If this is correct, coherence would thus work as a warrant for absolute normative consistency. 
assume that the latter is not trivially complete or absolutely inconsistent, since an absolutely inconsistent/trivially complete system of principles justifies any rule whatsoever.

However, consistency is a problematic property when it is predicated of a system of principles, if principles are regarded as defeasible norms. Indeed, if - as we mentioned before-the antecedent of a principle is not taken to be a sufficient condition (or to provide sufficient conditions) of its consequent, their proper formal representation should be something like:

$$
(p>O q)
$$

Here the corner ('>') is used to symbolize a defeasible conditional, one which neither allows modus ponens nor strengthening the antecedent. ${ }^{34}$ For this reason, a system of principles can contain [25] together with

$$
(p>P \sim q)
$$

with no inconsistency derivable from them, even for $p$-worlds, since $p$ does not constitute a sufficient condition for the provided consequence to follow.

It would seem, then, that the system of principles cannot be inconsistent by definition, since it is composed of defeasible normative standards. If it is so, however, it is not clear, from a logical point of view, how the relation of coherence between the system of principles and the system of rules should be framed. An option consists in regarding the principle as a "tentative confirmation" of the normative consequence provided by a certain set of rules. The more confirmed a certain normative solution provided by a rule is, the stronger appears the relation of coherence between underlying principles and such a rule. However, what is important to stress here is that the idea of competing confirmations presuppose inconsistencies of solutions within the system of rules. Coherence relations would thus be, or might be used, as tools liable to solve antinomies afflicting sets of rules. ${ }^{35}$

As a conclusion, coherence, as a theorized logical item, may be said to be a rather ambiguous combination of weak completeness, and possible absence of consistency and strong completeness of a system of rules regarding a non-trivially complete/non-absolutely inconsistent system of underlying principles.

\section{Bibliography}

A

Carlos E. ALCHOURRÓN, 1969: Logic of Norm and Logic of Normative Propositions. Logique et Analyse 12 (1969) 47: 242-268.

- 1993: Philosophical Foundations of Deontic Logic and the Logic of Defeasible Conditionals, in J. Meyer and R. Wieringa, Deontic Logic in Computer Science: Normative System Specification. Chichester - New York - Brisbane - Toronto - Singapore: Wiley \& Sons. 43-84.

Carlos E. ALCHOURRÓN \& Eugenio BULYGIN, 1971: Normative Systems. Wien - New York: Springer.

— 1988: Perils of Level Confusion in Normative Discourse. Rechtstheorie 19: 230-237.

Juan Pablo ALONSO, 2010: Interpretación de las normas y derecho penal. Buenos Aires: Editores del Puerto.

_ 2013: Principios jurídicos implícitos y coherencia. Doxa 36: 357-385.

Amalia AMAYA, 2007: Formal Models of Coherence and Legal Epistemology. Artificial Intelligence and Law 15: 429-447.

- 2015: The Tapestry of Reason. An Inquiry into the Nature of Coherence and its Role in Legal Argument. Oxford: Hart Publishing.

Michał ARASZKIEWICZ \& Jaromir ŠAVELKA (Eds.), 2013: Coherence. Insights from Philosophy, Jurisprudence and Artificial Intelligence. Dordrecht: Springer.

B 
Mathieu BEIRLAEN \& Christian STRASSER, 2013: Two Adaptive Logics of Norm-propositions. Journal of Applied Logic 11(2): 147-168.

Jeremy BENTHAM, 1970: Of Laws in General. Edited by H.L.A. Hart. London: Athlone Press.

Eugenio BULYGIN, 1995: Lógica deóntica, in Lógica. Enciclopedia Iberoamericana de Filosofía, Vol. 7. Eds. C.E. Alchourrón et al. Madrid: Trotta. 129-142.

$\mathrm{D}$

Ronald DWORKIN, 1978: Taking Rights Seriously. $2^{\text {nd }}$ edition. London: Duckworth.

Jordi FERRER BELTRÁN \& Giovanni Battista RATTI (Eds.), 2012: The Logic of Legal Requirements. Essays on Defeasibility. Oxford: Oxford University Press.

G

Riccardo GUASTINI, 2011: Interpretare e argomentare. Milan: Giuffrè.

$\mathrm{H}$

Jaap HAGE, 1997: Reasoning with Rules. An Essay on Legal Reasoning and Its Underlying Logic. Dordrecht: Kluwer Academic Publishers.

— 2005. Studies in legal Logic. Dordrecht: Springer.

Bengt HANSSON, 1969: An Analysis of Some Deontic Logics. Nous 3: 373-398.

Herbert L.A. HART, 1983: Essays in Jurisprudence and Philosophy. Oxford: Oxford University Press. Ingemar HEDENIUS, 1941: Om rätt och moral. Stockholm: Wahlström \& Widstrand.

George E. HUGHES \& David G. LONDEY, 1965: Elements of Formal Logic. London: Methuen.

Geoffrey HUNTER, 1971: Metalogic: An Introduction to the Metatheory of Standard First-Order Logic. Berkeley: University of California Press.

$\mathrm{M}$

Neil D. MacMORMICK, 1984: Coherence in Legal Justification, in Theory of Legal Science. Ed. A. Peczenik. Dordrecht: Reidel. 235-251.

$\mathrm{P}$

Aleksandet PECZENIK, 2008: On Law and Reason. $2^{\text {nd }}$ edition. Berlin: Springer.

$\mathrm{R}$

Nicholas RESCHER, 1973: The Coherence Theory of Truth. Oxford: Oxford University Press.

Jorge L. RODRÍGUEZ, 2002: Lógica de los sistemas jurídicos. Madrid: Centro de estudios políticos y constitucionales.

S

Frederick SCHAUER, 2009: Thinking like a Lawyer. A New Introduction to Legal Reasoning. Cambridge (Mass.): Harvard University Press.

W

Georg Henrik von WRIGHT, 1963: Norm and Action. A Logical Inquiry. London: Routledge \& Kegan Paul.

— 1983. Practical Reason. Philosophical Papers. Vol. I. Oxford: Basil Blackwell.

Anders WEDBERG, 1951: Some Problems in the Logical Analysis of Legal Science. Theoria 17: 246275.

$\check{Z}$

Berislav ŽARNIĆ \& Gabriela BAŠIĆ, 2014: Metanormative Principles and Norm Governed Social Interaction. Revus. Journal for Constitutional Theory \& Philosophy of Law (2014) 22: 105-120.

\section{Notes}

1 The formal analysis of coherence was introduced in Rescher (1973). In the domain of jurisprudence, among the major contributions to understanding the applications of the concept are MacCormick (1984) and Peczenick (2008). For recent surveys, see Araszkiewicz \& Šavelka (2013) and Amaya (2015).

2 For a formal approximation, see Amaya (2007) and Alonso (2013). 
3 See Hughes \& Londey (1965: 137-140).

4 Strictly speaking, a specification of the logical system under consideration should be added to each formula (e.g. ' $\mathrm{S}$ is absolutely consistent in logic $L$ iff $\exists p\left(p \notin \mathrm{Cn}_{L}(\mathrm{~S})\right)$ '). For the sake of simplicity we shall omit such a qualification here.

5 Along with these notions of completeness, which may be deemed semantic, several syntactic notions of completeness are conceivable, the most natural of which may be defined as follows: $\mathrm{S}$ is syntactically complete iff $\forall p(p \in \mathrm{Cn}(\mathrm{S}) \vee \sim p \in \mathrm{Cn}(\mathrm{S}))$ (see Hunter 1971: 33). This syntactic concept of completeness is closely related to the notion of normative completeness that will be presented above.

6 Jan Woleński suggested in private communication that the notion of post-completeness should be introduced into our analysis for the sake of clarity. Post-completeness is usually defined as follows: 'A system of logic is post-complete if every time we add to it a sentence unprovable in it, we obtain an inconsistent system.' We tend to reject such a suggestion, since we are not dealing here with completeness of the calculi (in meta-language), but with completeness of the object-language. At any rate, if we reinterpret post-completeness as a first-order notion, and drop the 'unprovable' connotation, we think it collapses into what we call strong completeness.

7 See Rodríguez (2002: 62 ff).

8 The most sophisticated model of normative systems - the one developed by Alchourrón \& Bulygin (1971: 98-101) - analyses in depth the relations between the two concepts regarding universes of cases characterized by different levels of specificity.

9 See Alchourrón \& Bulygin (1971). With different names, the distinction has been noticed by several authors, e.g. Bentham (1970), Hedenius (1941), Wedberg (1951), Hansson (1969), among others.

10 Von Wright (1963: 106).

11 Referring to the legal domain, Hart (1983: 329) observes 'There is frequent occasion for lawyers to describe what they might call the 'legal position' in relation to some subject without referring to the particular enactments or regulations or other sources of the relevant law, though of course it would be always understood that 'the legal position' thus described is that arising under the laws of a particular system, and a more accurate formulation would make this explicit by including such words as 'according to English law'.'

12 See Alchourrón (1969), Alchourrón \& Bulygin (1971).

13 See Alchourrón \& Bulygin (1988: 231).

14 The expression 'negation-norm' was coined by von Wright (1983: 130-209).

$15 \mathrm{We}$ prefer this terminology to the more common dichotomy weak vs. strong permission, since the latter seems to suggest that the strong/positive concept implies the weak/negative one, which is not necessarily the case.

16 See Alchourrón (1969), Alchourrón \& Bulygin (1971).

17 See Alchourrón (1993: 46).

18 One possible presentation is the following:

19 In the system LNP there is also an axiom and a rule of inference $\left(\mathrm{A}_{3}\right.$ and $\left.\mathrm{RI}_{2}\right)$ that are not found in the usual systems of deontic logic. This is, however, a minor difference; were it possible to add to LNP an analogous principle to the principle $P p \# \sim O \sim p, \mathrm{~A}_{3}$ and $\mathrm{RI}_{2}$ would be superfluous because they would be derivable from the other axioms and rules.

20 See Bulygin (1995: 135, 137).

21 Proof: by commutativity of conjunction in [5] we obtain $\sim\left(P^{+}{ }_{\mathrm{s}} p \wedge O_{\mathrm{s}} \sim p\right)$; by definition of conditional in the latter expression we get $P^{+}{ }_{\mathrm{s}} p \Rightarrow \sim O_{\mathrm{s}} \sim p$, and now, by introduction of conditional from [5] to this latter step we get $\sim\left(O_{\mathrm{s}} \sim p \wedge P^{+}{ }_{\mathrm{s}} p\right) \#\left(P^{+}{ }_{\mathrm{s}} p \Rightarrow \sim O_{\mathrm{s}} \sim p\right)$. By definition of conditional in [6] we obtain $\left(\sim O_{\mathrm{s}} \sim p\right.$ $\left.\Rightarrow P^{+}{ }_{\mathrm{s}} p\right)$, and now by introduction of conditional we get $\left(O_{\mathrm{s}} \sim p \vee P^{+}{ }_{\mathrm{s}} p\right) \Rightarrow\left(\sim O_{\mathrm{s}} \sim p \# P^{+}{ }_{\mathrm{s}} p\right)$.

22 One could think that if a contradiction implies everything, then does normative inconsistency not only imply normative completeness but also its negation, i.e. normative incompleteness. However, this would be an incorrect conclusion derived from the identification of norms and norm-propositions, since from the fact that a normative system is inconsistent what follows is that every norm belongs to it, not that every norm-proposition (i.e., the one affirming its incompleteness) is true about it.

23 If A leads to contradiction, then $\sim$ A.

24 For an alternative system of logic for norm-propositions where normative conflicts and gaps are avoided 'whenever possible', see Beirlaen \& Straßer (2013).

25 This is far from being an unproblematic assumption, but we cannot enter into such details now.

26 By 'hypotethical normative systems' we mean systems which are not made solely of unconditional (viz. categorical) norms. 
27 Within this compass, rules are usually understood as conditional normative sentences connecting certain operative facts to the deontic qualification of a state of affairs (according to our previous symbolization: $p \Rightarrow O q$ ).

28 Here we are indebted to Guastini (2011: $173 \mathrm{ff}$ ).

29 Here we are fundamentally following MacCormick's notion of coherence in law (see MacCormick (1984: 235-244)). We will not discuss other possible paths, like the ones developed by Jaap Hage (e.g., 1997, 2005).

30 On this point, see Alonso (2010: $155 \mathrm{ff}$ ).

31 See Guastini (2011: 188-189).

32115 N.Y. 506 (1889).

33 Indeed, such a rule is a defeasible and frequently defeated one, as is shown in Schauer (2009: 34).

34 For a recent survey on defeasible norms, see Ferrer Beltrán \& Ratti (2012).

35 This is an idea expressly maintained by Dworkin (1978: 27).

\section{References}

\section{Electronic reference}

Giovanni Battista Ratti and Jorge Luis Rodríguez, « On coherence as a formal property of normative systems », Revus [Online], 27 | 2015, Online since 20 August 2015, connection on 23 January 2016.

URL : http://revus.revues.org/3291 ; DOI : 10.4000/revus.3291

Bibliographical reference

Giovanni Battista Ratti and Jorge Luis Rodríguez, « On coherence as a formal property of normative systems », Revus, 27 | 2015,

\section{Authors}

\section{Giovanni Battista Ratti}

Professor in Legal Philosophy, Tarello Institute for Legal Philosophy, Department of Law, University of Genoa

\section{Battista Ratti}

Tarello Institute for Legal Philosophy

Via Balbi 30/18

16126 Genoa

Italy

E-mail: gbratti@unige.it

Jorge Luis Rodríguez

Professor in Legal Theory, Faculty of Law, National University of Mar del Plata

\section{$-4$}

Luis Rodríguez

Rawson 318

CP7600

Mar del Plata

Provincia de Buenos Aires

Argentina

E-mail: jorgerodriguez64@yahoo.com

\section{Copyright}

All rights reserved 


\section{Abstract}

The paper deals with the notions of consistency, completeness, and coherence within the normative domain. It investigates their mutual relations by singling out relative (to negation) and absolute consistency, weak, strong and trivial completeness, and three different functions of coherence (simplification, integration, and defeasance). The main upshot of the inquiry is that coherence may be regarded as a complex combination of weak completeness and possible absence of consistency and strong completeness of a system of rules regarding a non-trivially complete/non-absolutely inconsistent system of underlying principles.

\section{Index terms}

Keywords : consistency, completeness, coherence, conditionals, normative systems 